\title{
Peer Reviewers for the Journal of the American Board of Family Medicine in 2011
}

\section{Phillip Lupo, Jr., MLIS, and Anne Victoria Neale PhD, MPH}

The editors and staff of the fournal of the American Board of Family Medicine ( $7 A B F M)$ thank our peer reviewers for their time, expertise, and dedication to the $F A B F M$. Our peer-reviewing colleagues serve a vital role in building the evidence base of primary care. In 2011, 433 individuals provided peer reviews and advice regarding the suitability of articles for publication in the $7 A B F M$. Reviewers took an average of 26 days to submit their reviews.

\section{Top Reviewers}

We would like to recognize our top reviewers. Each of the manuscripts submitted to the $7 A B F M$ that undergo peer review is evaluated and scored by the editors; in the list below, peer reviewers with an asterisk next to their name are in the top $10 \%$

Conflict of interest: The authors are staff of the $7 A B F M$. (based on evaluation scores plus quantity of reviews) of all reviewers in 2011.

\section{Seeking New Reviewers!}

We also enjoy welcoming new peer reviewers into the fold. Peer reviewing is a vital service activity that helps authors, researchers, and reviewers themselves to improve their work and their dissemination messages. Resources for reviewers are available on the $\mathcal{A} A B F M$ website (http://jabfm.org/site/misc/PeerReviewers. xhtml). Reviewers are always free to decline a request or to indicate periods of unavailability. If you would like to volunteer to review for the $7 A B F M$, please download and complete the Peer Reviewer Form (which can be accessed at http://jabfm.org/site/misc/ PeerReviewForm.doc) and email it, along with your curriculum vitae, to jabfm@med.wayne.edu or fax to 313-577-9828. Thank you!

\author{
John Abramson \\ David A. Acosta \\ Alan M. Adelman \\ Adeleye I. Adeyemi \\ Nelia Afonso \\ Erin J. Aiello \\ Grace A. Alfonsi \\ Cynthia L. Alford \\ Ayoob Ali \\ Deborah Allen \\ William A. Alto* \\ Gail M. Amundson \\ J. Ellen Anderson \\ John S. Anderson \\ G L. Andriole \\ David Anthony \\ Christine Arenson \\ Bengt Arnetz \\ Forest W. Arnold
}

Jennifer M. Aro
Bruce Arroll
Liv Berit Augestad
Suzanne Bakken
David Bamberger
Mikhail Bargan
Vernon A. Barnes
Kirsten Barrett
Elizabeth Barrett-Connor
Dennis J. Baumgardner
Elizabeth G. Baxley
Max Bayard
John W. Beasley
Marvin Moe Bell
Ian M. Bennett*
Keisa Bennett
Alfred O. Berg*
George R. Bergus
Ethan Berke

Barbara A. Berman
Dawn Bielawski
Juliann Binienda
Hans Birk
Richard Birtwhistle*
Asit Biswas
Carol E. Blenning
George W. Bock
Thomas Bodenheimer
Hillary Bogner
John M. Boltri*
Robert C. Bowman
Patrick T. Bradshaw
David S. Brody
Carlos Brotons
Randall T. Brown
Sean T. Bryan
Frank Buntinx
Elizabeth A. Burns


Danielle Butler

Lucinda M. Buys

Walter L. Calmbach

Peter J. Carek

Ty Carroll

Barry L. Carter

William E. Cayley Jr

Prakash Chandra

Ranee Chatterjee

Rajesh Chauhan

William E. Chavey

Kaj S. Christensen

Elizabeth C. Clark

William D. Clark

Linda G. Clark

Richard D. Clover

Andreas Cohrssen*

Jack M. Colwill

Paul F. Crawford III

Jesse C. Crosson

Brian K. Crownover

Richard W. Crummer

Larry Culpepper*

Doyle M. Cummings

Timothy P. Daaleman

Jeanette M. Daly*

Elvan Daniels

Robert J. Darios

Alan K. David

Michael Z. David

Melinda M. Davis

Lauren DeAlleaume

Peter Del Fante

Chris Del Mar

M. Dianne Delva

Cristina Demian-Popescu

Satish P. Deshpande

Jennifer E. DeVoe

Vanessa Diaz

Lori M. Dickerson

L. Miriam Dickinson*

Lorena Dini

B Djavan

Sharon A. Dobie

Lisa Dodson

Mark P. Doescher*

Paul P. Doghramji

Susan Dovey

Marguerite Duane

S. Shevaun Duiker

Anne L. Dunlop
Clare A. Dykewicz

Charles B. Eaton

Milton Eder

Mark Edlund

Nancy C. Elder

Kristin K. Elliott

John W. Ely*

Shannon K. English

John W. Epling Jr

Bernard G. Ewigman

Lyle J. Fagnan

Gail Fahoome

Joshua J. Fenton*

Erik O. Fernandez y Garcia

Jeanne M. Ferrante*

Michael D. Fetters*

Terry S. Field

Edgar Figueroa*

Kenneth S. Fink*

Kevin Fiscella

Sue Flocke

Colleen T. Fogarty

Patricia Fontaine

Stuart Forman

E Foster

Chester H. Fox

Erin Fraher

Jennifer Frank

Christopher R. Frei

Linda M. French

John J. Frey III

Keith A. Frey

Ivar L. Frithsen

Sim S. Galazka

Mauro Gallitelli

Harsha V. Ganga

David Garr

Elizabeth Garrett

Thomas F. Gavagan

Stephen J. Genuis

Cynthia M. Geppert

Valerie J. Gilchrist

James M. Gill*

Dwenda K. Gjerdingen*

Adam O. Goldstein

Bharat Gopal

Rachel J. Gorwitz

J N. Goswami

Laura M. Gottlieb

Thomas Graf

John J. Graff
Judith A. Gravdal

Beverly B. Green*

Michael E. Green

Larry A. Green*

Jeffrey L. Greenwald

Jessica L. Greenwood

Michelle Greiver

Tomas L. Griebling

Michael Grover

Karen Gunning

David L. Hahn

Ricardo Hahn

Jacqueline R. Halladay

Jennifer L. Hamilton

Margaret A. Handley

Cynthia Haq

Diane M. Harper

Susan Hart-Hester

Robert L. Hatch

Sarah Hawley

Diana L. Heiman

Robin Helm

Nikhil Hemady

Eric Henley

Roschelle Heuberger

Vincent W. Hevern

John M. Hickner

Paul C. Hicks

Timothy P. Hofer

Neil Holland

Robert M. Hoover

J C. Hoyle

Brenda L. Hudson

William J. Hueston

Katherine Hurst

Andrew D. Innes

Henry R. Ivey, Jr.

Akila Iyer

Carlos Roberto Jaen*

Paul A. James

Muhammad A. Javed

Xiangyang Jiao

Masahito Jimbo*

Michel R. Joffres

Mark S. Johnson

Roger Jones

Linda S. Kahn

Shawn F. Kane

Rahul Kapur

Neil S. Kaye

Omar A. Khan 
Morteza Khodaee

Dana E. King

Diane A. Klein

Douglas J. Klein

Donald O. Kollisch

Richelle J. Koopman

Colin P. Kopes-Kerr

Neil Korsen

Claudia A. Kozinetz

David L. Kriegel II

Alex H. Krist*

Philip J. Kroth

Marieke Kruip

K Laederach

Forrest Lang

Robert Langan

Jason S. Lanham*

Frank Lawler*

Ross A. Lawrenson

Kenyatta Lee

Lawrence M. Leeman

Patricia M. Lenahan

Lawrence Leung

Barcey T. Levy*

You-ming Li

Deerajnath Lingutla

Stephen K. Liu

Maja-Lisa Loechen

Jason A. Logan

Everett E. Logue

Ghanshyam Lohiya

Lucy W. Loomis

David P. Losh*

Margaret M. Love

D A. Ludwick

James H. Lynch IV

Catherine L. Lysack

Paula K. Maasilta

Arch G. Mainous III

Joseph A. Mantey

Katherine Margo

Tsveti Markova

John Marley

Cara Marshall

Kate Martin

Luca Mascitelli

Victoria L. Mason

Samuel C. Matheny

Catherine J. Mathews

Dimitrios K. Matthaiou

Charles Maynard
Catherine McCarthy

Anna McCullough

Jane A. McElroy

John McKay

Juliet M. McKee

James F. McKenzie

Amy McQueen

David R. Mehr

Aaron Michelfelder

Baukje Miedema

Nelda Mier*

Virginia Miller

Will Miller

James W. Mold

Christopher P. Morley

Karen Muchowski

Marlon P. Mundt

William J. Murdoch

Joseph M. Murley

David M. Murray

Zsolt J. Nagykaldi*

Jatinder Narang

Raj Narayan

Richard Neill

Lynne S. Nemeth

Warren P. Newton

Susan L. Norris

Mary Patricia Nowalk

Jim Nuovo

Maeve O'Beirne

Alane B. O'Connor

Catherine A. O'Donnell

Cathleen O'Farrell

Thomas R. O’Neill*

Kara Odom Walker

$\mathrm{M}$ N. Oliver

Steven M. Ornstein

A John Orzano

Lynda J. Owen

Joel Paris

Neela K. Patel

Donald Pathman*

Dale A. Patterson

Valory N. Pavlik*

Linda L. Pederson

Allen L. Pelletier

Kevin A. Peterson

P D. Phatak

William R. Phillips

Margaret B. Planta

Charles D. Ponte
John H. Porcerelli

Shailendra Prasad*

David Price

James C. Puffer

Howard K. Rabinowitz*

Francine Rainone

Mithun S. Rajput

Kalyanakrishnan Ramakrishnan

Stephen Ratcliffe*

Joanne L. Reeve

Jo Marie Reilly

Michael D. Reis

Carol Rheaume

Karin Rhodes

Robert L. Rhyne

Karen Richardson-Nassif

Peter A. Rives

Charo Rodriguez*

Hector P. Rodriguez

Michelle A. Roett

Martin Roland

Elaine Rose

Thomas C. Rosenthal

Steven E. Roskos

Kenneth D. Royal

Robert S. Rudin

Mack T. Ruffin* IV

Terry S. Ruhl

Monika M. Safford

Enrique Sanchez Valdivieso

Robert B. Saper

Judith A. Savageau*

Barry G. Saver

Amir M. Sayem

Ellen I. Schafheutle

Maryjean Schenk

Joseph E. Scherger

Guido Schmiemann

Loes M. Schouten

Sarina Schrager*

Gerry K. Schwalfenberg

Kendra Schwartz

J. Paul Seale

Dean A. Seehusen*

Egambaram Senthilvel

Richard K. Severson

Javad Shahidi

Eric K. Shaw

Angela J. Shepherd

Ruth S. Shim

Scott A. Shipman 
Navkiran K. Shokar

Matthew W. Short

Leonard A. Shulman

Pesach Shvartzman

Hugh Silk

Mindy A. Smith

Douglas Smucker

Leif I. Solberg

William Spears

Ra Nae Stanton

Jeffrey A. Stearns

Shane P. Stenner

CDR Mark B. Stephens

Margaret Stineman

Stefan Stoerk

Gregg D. Stoner

Steven D. Stovitz

Gregory Strayhorn

George D. Strelioff

Brian Strom

Andrew L. Sussman*

John E. Sutherland

Paul R. Swank*

David E. Swee

Jens Søndergaard
Hazel Tapp

Nicole Tartaglia

Chip A. Taylor

Peter G. Teichman

Chris M. Terpening

Joshua Tessier

Jeffrey Tiemstra

Katherine H. Tkaczuk

Matthew E. Ulven

Carole C. Upshur

Henriette E. van der Horst

Liset van Dijk

Chris van Weel

Annapia Verri

Anthony J. Viera

Daniel C. Vinson

Eric Wall*

Judith M.E. Walsh

Richard C. Wasserman

Barry D. Weiss

David P. Weller

James J. Werner*

David R. West*

Randy K. Wexler

Lauren Whetstone
Brett White

Philip S. Whitecar

Joanne E. Wilkinson

Paul S. Williamson

Calvin L. Wilson

Stephen A. Wilson

Daniel K. Winstead

Cathy Wong

Laurie Woodard

William M. Woodhouse

W. Michael Woods

Tsu-Yin $\mathrm{Wu}$

Imam Xierali

Jinping $\mathrm{Xu}^{*}$

Mark J. Yaffe

Barbara P. Yawn

Atif Zafar

Min Zhang

Therese Zink

Stephen J. Zyzanski

*Top reviewer in 2011 (based on quantity and quality of peer reviews written). 